\title{
A New Numerical Method of Particular Solutions for Inhomogeneous Burgers' Equation
}

\author{
Huantian Xie, ${ }^{1,2}$ Dingfang $\mathrm{Li}^{2}$ and Feng $\mathrm{Li}^{1}$ \\ ${ }^{1}$ School of Science, Linyi University, Linyi 276005, China \\ ${ }^{2}$ School of Mathematics and Statistics, Wuhan University, Wuhan 430072, China \\ Correspondence should be addressed to Huantian Xie; xht0539@qq.com
}

Received 9 May 2013; Accepted 28 June 2013

Academic Editor: Fazal M. Mahomed

Copyright (c) 2013 Huantian Xie et al. This is an open access article distributed under the Creative Commons Attribution License, which permits unrestricted use, distribution, and reproduction in any medium, provided the original work is properly cited.

Based on the finite difference scheme in time, the method of particular solutions using radial basis functions is proposed to solve one-dimensional time-dependent inhomogeneous Burgers' equations. Two numerical examples with good accuracy are given to validate the proposed method.

\section{Introduction}

In this paper we consider the one-dimensional nonlinear evolutionary partial differential equation

$$
\frac{\partial u(x, t)}{\partial t}+u(x, t) \frac{\partial u(x, t)}{\partial x}-\frac{1}{R} \frac{\partial^{2} u(x, t)}{\partial x^{2}}=f(x, t) \text {. }
$$

The corresponding homogeneous equation

$$
\frac{\partial u(x, t)}{\partial t}+u(x, t) \frac{\partial u(x, t)}{\partial x}-\frac{1}{R} \frac{\partial^{2} u(x, t)}{\partial x^{2}}=0
$$

was first introduced by Bateman [1] who considered its steady state solutions. Later, Burgers [2, 3] treated it as a mathematical model for free turbulence, and subsequently this equation is widely referred to as Burgers' equation. Burgers' equation can model several physical phenomena such as traffic, shock waves, and continuous stochastic processes. It can also be used to test various numerical algorithms. Due to its wide range of applicability, several researchers have been interested in the properties of its solution.

Burgers' equation has been solved analytically for a restricted set of arbitrary initial and boundary conditions [4, 5]. Benton and Platzman [6] surveyed about 35 distinct exact solutions of the one-dimensional Burgers-like equations and their classifications. It is well known that the exact solution of Burgers' equation can only be computed for restricted values of $R$. Therefore, various numerical methods were employed to obtain the solution of Burgers' equation. It is not our purpose to exhaust all these numerical schemes. Nevertheless, the solution methodologies commonly fall into the following classes: finite difference method (FDM), finite element method (FEM), and spectral methods. A survey of these techniques is given in $[7,8]$. The previous numerical methods all depend on the mesh of the studied domain [912].

To alleviate the difficulty of mesh generation, various meshless techniques have been introduced during the past two decades. In a meshless (meshfree) method, a set of scattered nodes are selected in the computational domain. Meshless schemes include the method of fundamental solutions (MFS) [13], the method of particular solutions (MPS) [14-17], the element-free Galerkin method [18], local point interpolation [12], and boundary knot method [19]. It is known that the MFS is a boundary-type meshless method which is highly accurate for solving homogeneous equations if the fundamental solution of the given differential operator is known [20]. However, the fundamental solution of a given differential equation is not always available and often very difficult to derive. The ill-conditioning of the matrix resulting from the formulation of using the MFS and the location of source points are still outstanding research problems. 
To extend the MFS to inhomogeneous equations or timedependent problems, the MPS has been introduced to evaluate the particular solution of the given differential equation. Since the particular solution is not unique, there is a rich variety of numerical techniques developed for this purpose.

Radial basis functions (RBFs), polynomial functions, trigonometric functions, and so forth [20-24], have been employed as the basis functions to approximate the particular solutions for the given differential equation. Once a particular solution has been evaluated, the given inhomogeneous equations can be reduced to the homogeneous equation. The original differential equation can be recovered by adding the homogeneous solution and the particular solution. This is a two-stage numerical scheme and is a well-known procedure for solving linear partial differential equations.

In general, the fundamental solution can be viewed as a special type of particular solution. When the inhomogeneous term is replaced by the delta function, a particular solution becomes a fundamental solution. The main idea of the MFS is that a fundamental solution satisfies the homogeneous equation inside the domain, and one only needs to enforce the fundamental solution on the boundary conditions to obtain the solution of the given homogeneous problem. Motivated by a similar idea, the particular solution can be used to solve inhomogeneous problems; that is, since the particular solution satisfies the given inhomogeneous equation through the domain without satisfying the boundary conditions, one only needs to impose the boundary conditions to obtain the solution of the given inhomogeneous problem.

It is the purpose of this paper to extend the MPS to a one-stage numerical scheme for solving one-dimensional time-dependent Burgers' equations through the use of RBFs and then solve the time-dependent problems without the need of a two-stage numerical scheme [25] for obtaining homogeneous solution.

\section{The Method of Particular Solutions}

Consider the following boundary value problem:

$$
\begin{aligned}
\Delta u(\mathbf{x})=f(\mathbf{x}), & \mathbf{x} \in \Omega, \\
B u(\mathbf{x})=b(\mathbf{x}), & \mathbf{x} \in \Gamma,
\end{aligned}
$$

where $\Delta$ and $B$ are the Laplace operator and boundary differential operators, respectively, $\Omega \subseteq \mathbb{R}^{d}$ is the solution domain, $\Gamma$ is its boundary, and $f(\mathbf{x})$ and $b(\mathbf{x})$ are given functions.

Approximate $f(\mathbf{x})$ by a finite series of RBFs $\left\{\phi_{j}\right\}_{1}^{N}$ through interpolation, and interpolants $\widehat{f}_{n}(\mathbf{x})$ to $f(\mathbf{x})$ can be constructed as

$$
f(\mathbf{x}) \simeq \widehat{f}_{N}(\mathbf{x})=\sum_{j=1}^{N} \lambda_{j} \phi\left(r_{j}\right)
$$

in which $r_{j}=\left\|\mathbf{x}-\mathbf{x}_{j}\right\|$ is Euclidean distance, $\left\{\mathbf{x}_{j}\right\}_{1}^{N}$ is a set of interpolation points, and the real coefficients $\left\{\lambda_{j}\right\}_{1}^{N}$ are to be determined by solving

$$
\sum_{j=1}^{N} \lambda_{j} \phi\left(r_{j}\right)=f\left(\mathbf{x}_{k}\right), \quad 1 \leq k \leq N
$$

if the $n \times n$ real coefficient matrix $A_{\phi}=\left(\phi\left(r_{k j}\right)\right)$ is positive definite.

Therefore, from (6) an approximate particular solution $u(\mathbf{x})$ to (3) is given by

$$
u(\mathbf{x}) \simeq \sum_{j=1}^{N} \lambda_{j} \Phi\left(r_{j}\right),
$$

where $\Phi\left(r_{j}\right)$ is obtained analytically by solving

$$
\Delta \Phi\left(r_{j}\right)=\phi\left(r_{j}\right) .
$$

If we impose $u(\mathbf{x})$ in (7) to satisfy the governing equation in (3) and boundary conditions in (4), then $u(\mathbf{x})$ becomes an approximate solution of the original partial differential equations (3)-(4). To be more specific, we have

$$
\begin{aligned}
& \sum_{j=1}^{N} \lambda_{j} \phi\left(r_{j}\right)=f\left(\mathbf{x}_{k}\right), \quad \mathbf{x}_{k} \in \Omega, \\
& \sum_{j=1}^{N} \lambda_{j} B \Phi\left(r_{j}\right)=b\left(\mathbf{x}_{k}\right), \quad \mathbf{x}_{k} \in \Gamma .
\end{aligned}
$$

For the numerical implementation, we let $\left\{\mathbf{x}_{k}\right\}_{1}^{n_{I}}$ be the interior points, $\left\{\mathbf{x}_{k}\right\}_{n_{I}+1}^{N}$ the boundary points, and $N=n_{I}+n_{B}$. From (9) we have

$$
\sum_{j=1}^{N} \lambda_{j} \phi\left(r_{j}\right)=f\left(\mathbf{x}_{k}\right), \quad 1 \leq k \leq n_{I},
$$

$$
\sum_{j=1}^{N} \lambda_{j} B \Phi\left(r_{j}\right)=b\left(\mathbf{x}_{k}\right), \quad n_{I}+1 \leq k \leq N
$$

The above system of equations can be easily solved using a standard matrix solver. Once the $\left\{\lambda_{j}\right\}_{1}^{N}$ are determined, the approximate particular solution becomes the approximate solution $u(\mathbf{x})$ of (3)-(4); that is,

$$
u(\mathbf{x})=\sum_{j=1}^{N} \lambda_{j} \Phi\left(r_{j}\right) .
$$

Note that an accurate approximation of the particular solution $u(\mathbf{x})$ depends on the appropriate choice of radial basis function $\phi$. In the RBF literature $[20,26]$, some of the globally defined RBFs are only conditionally positive definite [26]. The unique solvability of the interpolation problem can be obtained by adding a polynomial term to the interpolation (5), giving

$$
f(\mathbf{x}) \simeq \widehat{f}_{n}(\mathbf{x})=\sum_{j=1}^{N} \lambda_{j} \phi\left(r_{j}\right)+\sum_{k=1}^{K} \mu_{k} p_{k}(\mathbf{x}),
$$


along with the constraints

$$
\sum_{j=1}^{N} \lambda_{j} p_{k}\left(\mathbf{x}_{j}\right)=0, \quad 1 \leq k \leq K,
$$

where $\left\{p_{k}\right\}_{1}^{K}$ is a basis of $\mathscr{P}_{m-1}$, the space of $d$-variate polynomials of order not exceeding $m-1$, and

$$
K=\left(\begin{array}{c}
m-1+d \\
d
\end{array}\right)
$$

is the dimension of $\mathscr{P}_{m-1}$.

There are many types of globally defined RBFs [20], and the most popular RBFs are

Inverse multiquadric (IMQ),

$$
\phi(r)=\left(r^{2}+c^{2}\right)^{q / 2}, \quad q<0, c>0
$$

Multiquadric (MQ),

$$
\phi(r)=\left(r^{2}+c^{2}\right)^{q / 2}, \quad q \in \mathbb{R}^{+} \backslash 2 \mathbb{Z}, c>0, m>\left[\frac{q}{2}\right],
$$

Gaussian (G),

$$
\varphi(r)=e^{-c r^{2}}, \quad c>0 .
$$

Polyharmonic (PH),

$$
\phi(r)=r^{q}, \quad q \in \mathbb{R}^{+} \backslash 2 \mathbb{Z}, c>0, m>\left[\frac{q}{2}\right] .
$$

Polyharmonic (PH),

$$
\phi(r)=r^{q} \log (r), \quad q \in 2 \mathbb{Z}, m>\frac{q}{2} .
$$

\section{Methodology}

In order to solve such whole-space problems by numerical methods, we limit our consideration to a finite subdomain $[a, b]$. In other words, function $u(x, t)$ satisfies the following general nonlinear one-dimensional time-dependent Burgers' equation:

$$
\begin{array}{r}
\frac{\partial u(x, t)}{\partial t}=\frac{1}{R} \frac{\partial^{2} u(x, t)}{\partial x^{2}}-u(x, t) \frac{\partial u(x, t)}{\partial x}+f(x, t) \\
(x, t) \in[a, b] \times[0, T],
\end{array}
$$

with the initial condition

$$
u(x, 0)=g(x), \quad x \in[a, b],
$$

and the Dirichlet boundary condition

$$
u(a, t)=p(t), \quad u(b, t)=q(t), \quad t \in[0, T],
$$

where $R>0$ is interpreted as the Reynolds number, and $1 / R$ is the kinematic viscosity, and $f(x, t), g(x), p(t)$, and $q(t)$ are known functions.
In the following section, a generalized trapezoidal method ( $\theta$-method) is used to approximate the time derivative in (20). Let $\tau=t^{n+1}-t^{n}$ be the time step and $t^{n}=n \tau$. For any $t^{n} \leq t \leq t^{n+1}$ and $0 \leq \theta \leq 1, u(x, t)$ can be approximated as follows:

$$
\begin{aligned}
& u(x, t) \simeq \theta u\left(x, t^{n+1}\right)+(1-\theta) u\left(x, t^{n}\right), \\
& f(x, t) \simeq \theta f\left(x, t^{n+1}\right)+(1-\theta) f\left(x, t^{n}\right) .
\end{aligned}
$$

Then,

$$
\begin{gathered}
\Delta u(x, t) \simeq \theta \Delta u\left(x, t^{n+1}\right)+(1-\theta) \Delta u\left(x, t^{n}\right), \\
\frac{\partial u(x, t)}{\partial t} \simeq \frac{u\left(x, t^{n+1}\right)-u\left(x, t^{n}\right)}{\tau},
\end{gathered}
$$

where $\Delta=\partial^{2} / \partial x^{2}$. For simplicity, we denote $u^{n} \equiv$ $u\left(x, t^{n}\right), u_{x}^{n} \equiv \partial u\left(x, t^{n}\right) / \partial x$, and $f^{n} \equiv f\left(x, t^{n}\right)$. Substituting (23)-(24) into (20)-(22), we obtain the following equation:

$$
\begin{aligned}
\frac{u^{n+1}-u^{n}}{\tau}= & \theta\left[\frac{1}{R} \Delta u^{n+1}-u^{n+1} u_{x}^{n+1}+f^{n+1}\right] \\
& +(1-\theta)\left[\frac{1}{R} \Delta u^{n}-u^{n} u_{x}^{n}+f^{n}\right] .
\end{aligned}
$$

Rewrite the previous equation as follows:

$$
\begin{aligned}
u^{n+1}- & \theta \tau\left[\frac{1}{R} \Delta u^{n+1}-u^{n+1} u_{x}^{n+1}+f^{n+1}\right] \\
& =u^{n}+(1-\theta) \tau\left[\frac{1}{R} \Delta u^{n}-u^{n} u_{x}^{n}+f^{n}\right] .
\end{aligned}
$$

Then,

$$
\begin{aligned}
\Delta u^{n+1}= & \frac{R}{\theta \tau} u^{n+1}+R u^{n+1} u_{x}^{n+1}-R f^{n+1} \\
& -\frac{R}{\theta \tau} u^{n}-\frac{R(1-\theta)}{\theta}\left[\frac{1}{R} \Delta u^{n}-u^{n} u_{x}^{n}+f^{n}\right] .
\end{aligned}
$$

Assume that $u^{n+1}(x)$ is a sought solution to the elliptic PDE. We can represent the right hand side of (27) as a function $F(x)$. This means that (27) is a standard Poisson-type differential equation

$$
\Delta u^{n+1}=F(x) .
$$

Therefore, if the fictitious function $F(x)$ is known, (27) is equivalent to the Poisson-type equation (28) under the same boundary conditions.

Uniformly choose $N-2$ collocation points $\left\{x_{k}\right\}_{2}^{N-1}$ in the interior of domain $[a, b]$ and two boundary points $x=a$ and $x=b$. For implementation, let $x_{1}=a, x_{N}=b$. Approximating the function $F(x)$ by RBFs $\left\{\phi_{j}\right\}_{1}^{N}$, we have

$$
F(x) \simeq \sum_{j=1}^{N} \lambda_{j}^{n+1} \phi_{j}\left(r_{k j}\right), \quad k, j=1,2, \ldots, N,
$$


where $r_{k j}=\left\|\left(x_{k}-x_{j}\right)^{2}\right\|$. Then we can approximate $u(x)$ at time step $n+1$ as follows:

$$
u^{n+1}(x) \simeq \sum_{j=1}^{N} \lambda_{j}^{n+1} \Phi_{j}\left(r_{j}\right)
$$

where $\Phi_{j}(x)$ is obtained by solving

$$
\Delta \Phi(x)=\phi(x) .
$$

We use two RBFs, namely, IMQ and PH, where

$$
\phi_{j}(x)=\frac{1}{\sqrt{\left(r_{j}^{2}+c^{2}\right)^{3}}}, \quad \phi_{k}(x)=r_{k}^{3} .
$$

It is easy to obtain the following $\Phi(x)$ :

$$
\Phi_{j}(x)=\frac{1}{c^{2}} \sqrt{r_{j}^{2}+c^{2}}, \quad \Phi_{k}(x)=\frac{1}{20} r_{k}^{5} .
$$

Note that (27) is a recursion formula, and we can solve each elliptic PDE step by step starting with initial condition (21). As is well known, it is difficult to obtain an accurate numerical derivative from scattered data. Therefore, we choose $\theta=1$ in our method in order to avoid evaluating $\Delta u^{n}$ in (27). In this case, we can reformulate (27) as follows:

$$
u^{n+1}-\tau\left[\frac{1}{R} \Delta u^{n+1}-u^{n+1} u_{x}^{n+1}\right]=u^{n}+\tau f^{n+1} .
$$

The nonlinear term $u^{n+1} u_{x}^{n+1}$ is linearized as follows:

$$
u^{n+1} u_{x}^{n+1}=u^{n+1} u_{x}^{n}+u^{n} u_{x}^{n+1}-u^{n} u_{x}^{n} .
$$

Substituting (35) into (34) and rearranging, we obtain

$$
\begin{aligned}
u^{n+1}- & \tau\left[\frac{1}{R} \Delta u^{n+1}-u^{n+1} u_{x}^{n}-u^{n} u_{x}^{n+1}\right] \\
& =u^{n}+\tau u^{n} u_{x}^{n}+\tau f^{n+1} .
\end{aligned}
$$

Write (30) together with boundary condition (22) in matrix form

$$
[u]^{n}=A[\lambda]^{n},
$$

where $[u]^{n}=\left[u_{1}^{n}, u_{2}^{n}, \ldots, u_{N}^{n}\right],[\lambda]^{n}=\left[\lambda_{1}^{n}, \lambda_{2}^{n}, \ldots, \lambda_{N}^{n}\right]$, and $A=\left[a_{k j}\right], 1 \leq k, j \leq N$. There are $N-2$ internal points and 2 boundary points. The $N \times N$ matrix $A$ can be split into $A=A_{d}+A_{b}$, where

$$
\begin{gathered}
A_{d}= \begin{cases}\left(a_{k j}\right) & \text { if } 2 \leq k \leq N-1,1 \leq j \leq N \\
a_{k j}=0 & \text { otherwise, }\end{cases} \\
A_{b}= \begin{cases}\left(a_{k j}\right) & \text { if } k=1, N, 1 \leq j \leq N \\
a_{k j}=0 & \text { otherwise. }\end{cases}
\end{gathered}
$$

Applying this to the domain points and boundary points, (37) and (22) can be reformulated in the following matrix form:

$$
\begin{aligned}
& \left\{\mathbf{A}_{d}-\tau \varepsilon \nabla^{2} \mathbf{A}_{d}\right. \\
& \left.+\tau\left[\operatorname{diag}\left(\mathbf{A}_{d}[\lambda]^{n}\right) \nabla \mathbf{A}_{d}+\operatorname{diag}\left(\nabla \mathbf{A}_{d}[\lambda]^{n}\right) \mathbf{A}_{d}\right]+\mathbf{A}_{b}\right\}[\lambda]^{n+1} \\
& \quad=\mathbf{A}_{d}[\lambda]^{n}+\tau\left(\mathbf{A}_{d}[\lambda]^{n}\right) \cdot *\left(\nabla \mathbf{A}_{d}[\lambda]^{n}\right)+[F]^{n+1}
\end{aligned}
$$

where $\nabla$ is the gradient differential operator, $\operatorname{diag}\left(\mathbf{A}_{d}[\lambda]^{n}\right)$ is a diagonal matrix with $\mathbf{A}_{d}[\lambda]^{n}$ as its main diagonal, $[F]^{n+1}=$ $\left[p^{n+1}, \tau f_{2}^{n+1}, \ldots, \tau f_{N-1}^{n+1}, q^{n+1}\right]^{T}$, and the accent “**" means component by component multiplication of two vectors.

\section{Numerical Results}

Two different problems are used to test the accuracy of our method. In order to evaluate the numerical errors, we adopt three kinds of errors defined by

$$
\begin{aligned}
L_{\infty} & =\max _{j}\left|u_{j}-U_{j}\right|, \\
L_{2} & =\sqrt{\sum_{j=1}^{N}\left|u_{j}-U_{j}\right|^{2}}, \\
\text { RMS } & =\sqrt{\frac{1}{N} \sum_{j=1}^{N}\left|u_{j}-U_{j}\right|^{2}},
\end{aligned}
$$

where $u_{j}=u\left(x_{j}, T\right)$ is the exact analytical solution, and $U_{j}$ is the numerical solution of $u_{j}$.

Example 1. Consider the following nonlinear onedimensional time-dependent Burgers' equation with a large Reynolds number $R=10000$, in the square domain $[0,2 \pi]$,

$$
\begin{gathered}
\frac{\partial u(x, t)}{\partial t}+u(x, t) \frac{\partial u(x, t)}{\partial x}-\frac{1}{R} \frac{\partial^{2} u(x, t)}{\partial x^{2}} \\
=-\frac{1}{2} \sin (2 x) e^{-2 t / R},
\end{gathered}
$$

with the initial condition

$$
u(x, 0)=\cos (x),
$$

and the boundary condition

$$
u(x, t)=u(x+2 \pi, t) .
$$

The analytical solution is given as

$$
u(x, t)=\cos (x) e^{-t / R} .
$$

We choose two RBFs, namely, IMQ and PH, as defined in (32). The $L_{\infty}, L_{2}$, and root-mean-square (RMS) errors for our 
TABLE 1: $L_{\infty}, L_{2}$, and RMS errors, with $\tau=0.1, d_{x}=\pi / 10$, and $x \in[0,2 \pi]$.

\begin{tabular}{|c|c|c|c|c|c|c|}
\hline \multirow{2}{*}{$t$} & \multicolumn{2}{|c|}{$L_{\infty}$} & \multicolumn{2}{|c|}{$L_{2}$} & \multicolumn{2}{|c|}{ RMS } \\
\hline & IMQ & $\mathrm{PH}$ & IMQ & $\mathrm{PH}$ & IMQ & $\mathrm{PH}$ \\
\hline 0.5 & $9.6592 \times 10^{-6}$ & $7.7103 \times 10^{-3}$ & $1.3706 \times 10^{-5}$ & $8.2398 \times 10^{-3}$ & $3.0648 \times 10^{-6}$ & $1.8425 \times 10^{-3}$ \\
\hline 1.0 & $9.9640 \times 10^{-6}$ & $1.2424 \times 10^{-2}$ & $1.5692 \times 10^{-5}$ & $1.7040 \times 10^{-2}$ & $3.5088 \times 10^{-6}$ & $3.8102 \times 10^{-3}$ \\
\hline 1.5 & $9.9985 \times 10^{-6}$ & $1.5138 \times 10^{-2}$ & $1.4249 \times 10^{-5}$ & $2.3674 \times 10^{-2}$ & $3.1863 \times 10^{-6}$ & $5.2937 \times 10^{-3}$ \\
\hline 2.0 & $1.7471 \times 10^{-6}$ & $2.7952 \times 10^{-2}$ & $2.7217 \times 10^{-5}$ & $4.0602 \times 10^{-2}$ & $6.0859 \times 10^{-6}$ & $9.0788 \times 10^{-3}$ \\
\hline
\end{tabular}

TABLE 2: $L_{\infty}, L_{2}$, and RMS errors, with $\tau=0.1, d_{x}=0.05$, and $x \in[0,1]$.

\begin{tabular}{|c|c|c|c|c|c|c|}
\hline \multirow{2}{*}{$t$} & \multicolumn{2}{|c|}{$L_{\infty}$} & \multicolumn{2}{|c|}{$L_{2}$} & \multicolumn{2}{|c|}{ RMS } \\
\hline & IMQ & $\mathrm{PH}$ & IMQ & $\mathrm{PH}$ & IMQ & $\mathrm{PH}$ \\
\hline 0.5 & $1.0490 \times 10^{-4}$ & $6.2473 \times 10^{-3}$ & $3.8990 \times 10^{-4}$ & $1.9479 \times 10^{-2}$ & $5.5140 \times 10^{-5}$ & $2.7547 \times 10^{-3}$ \\
\hline 1.0 & $2.6814 \times 10^{-4}$ & $1.0352 \times 10^{-2}$ & $1.0921 \times 10^{-3}$ & $3.2281 \times 10^{-2}$ & $1.5445 \times 10^{-4}$ & $4.5652 \times 10^{-2}$ \\
\hline 1.5 & $7.1806 \times 10^{-4}$ & $1.7210 \times 10^{-2}$ & $2.8510 \times 10^{-3}$ & $5.3690 \times 10^{-2}$ & $4.0320 \times 10^{-4}$ & $7.5929 \times 10^{-3}$ \\
\hline 2.0 & $1.9485 \times 10^{-3}$ & $2.8760 \times 10^{-2}$ & $7.8048 \times 10^{-3}$ & $8.9872 \times 10^{-2}$ & $1.1038 \times 10^{-3}$ & $1.2710 \times 10^{-2}$ \\
\hline
\end{tabular}

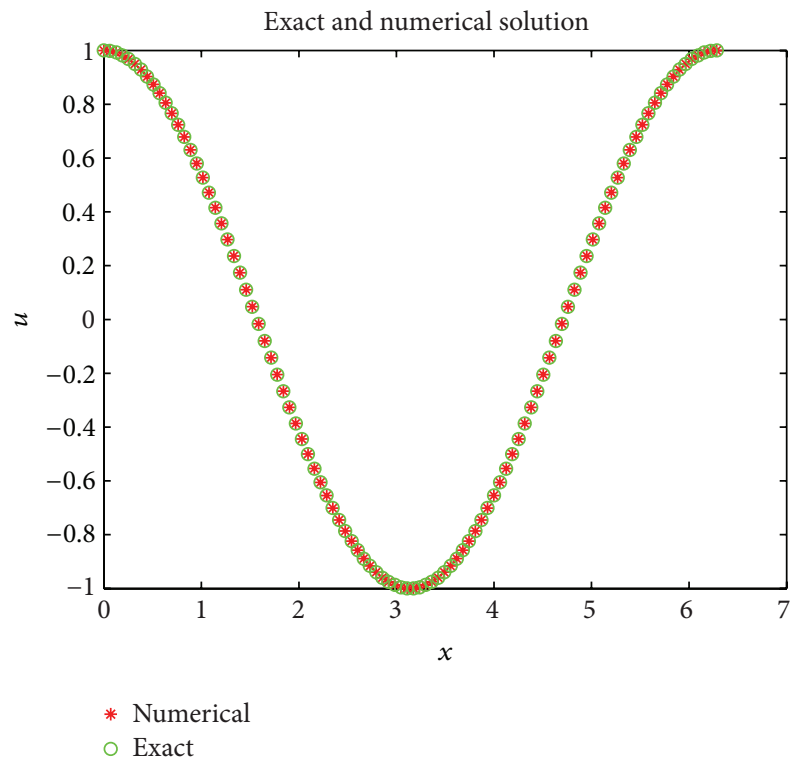

FIGURE 1: Analytical and numerical solution at $t=2$, with IMQ as RBF, $\tau=0.1, d_{x}=\pi / 10$, and $x \in[0,2 \pi]$, for Example 1 .

numerical solutions are shown in Table 1 for $t=0.5,1,1.5$, and 2 . It can be seen that the accuracy of the method using IMQ is much higher than that using PH.

The graph of the analytical and estimated solutions for $t=$ 2 is shown in Figure 1. The absolute error graph is shown in Figure 2. We also show the space-time graph of the estimated solution in Figure 3.

Example 2. We consider the second-order nonlinear Burgers' equation

$$
\frac{\partial u(x, t)}{\partial t}+u(\mathrm{x}, t) \frac{\partial u(x, t)}{\partial x}-\frac{1}{R} \frac{\partial^{2} u(x, t)}{\partial x^{2}}=f(x, t)
$$

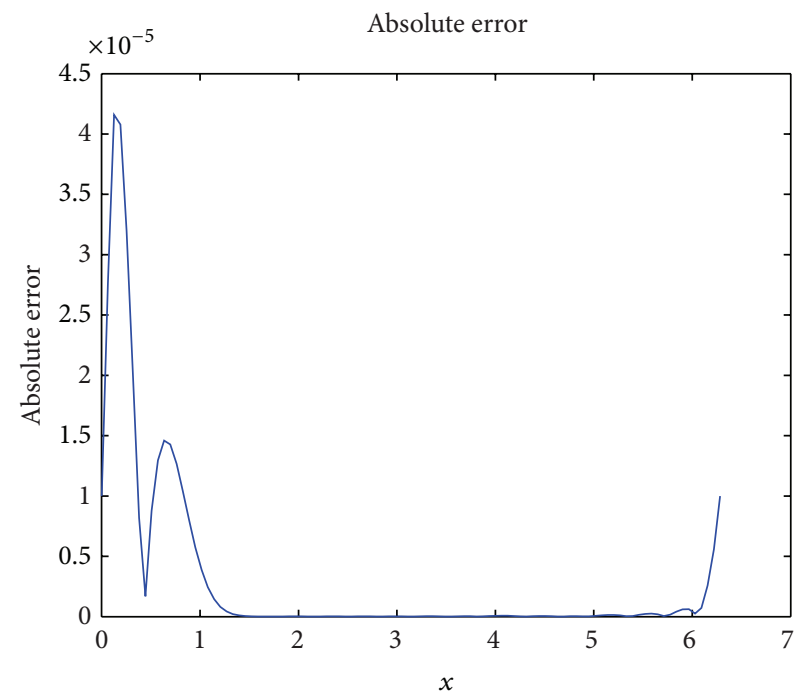

FIGURE 2: Absolute error at $t=2$, with IMQ as RBF, $\tau=0.1, d_{x}=$ $\pi / 10$, and $x \in[0,2 \pi]$, for Example 1 .

where $f(x, t)$ depends upon the exact solution of (45) as follows:

$$
u(x, t)=\left[\sin (2 \pi x)+\frac{1}{2} \sin (\pi x)\right] e^{t}
$$

We take the required initial and boundary functions from the exact solution in the domain [0,1]. Similar to Example 1, we also choose two RBFs, namely, IMQ and PH, as shown in (32). In Table 2, we compute the $L_{\infty}, L_{2}$ errors and RMS errors at $t=0.5,1,1.5$, and 2 , with the Reynolds number $R=0.001$. It indicates that the case of IMQ also has higher accuracy than the case of PH. In Figure 4, we draw the graph of the analytical and estimated solutions, and in Figure 5 the absolute error graph is shown. Figures 4 and 5 are shown at $t=2$. The spacetime graph of the numerical solution is shown in Figure 6. 


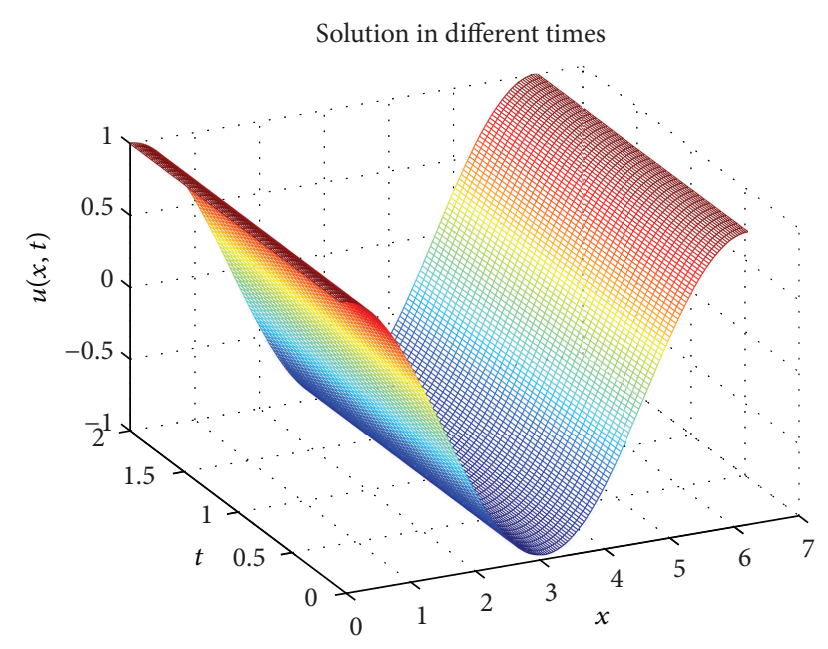

FIGURE 3: Space-time graph of the solution up to $t=2$, with IMQ as RBF, $\tau=0.1, d_{x}=\pi / 10$, and $x \in[0,2 \pi]$, for Example 1 .

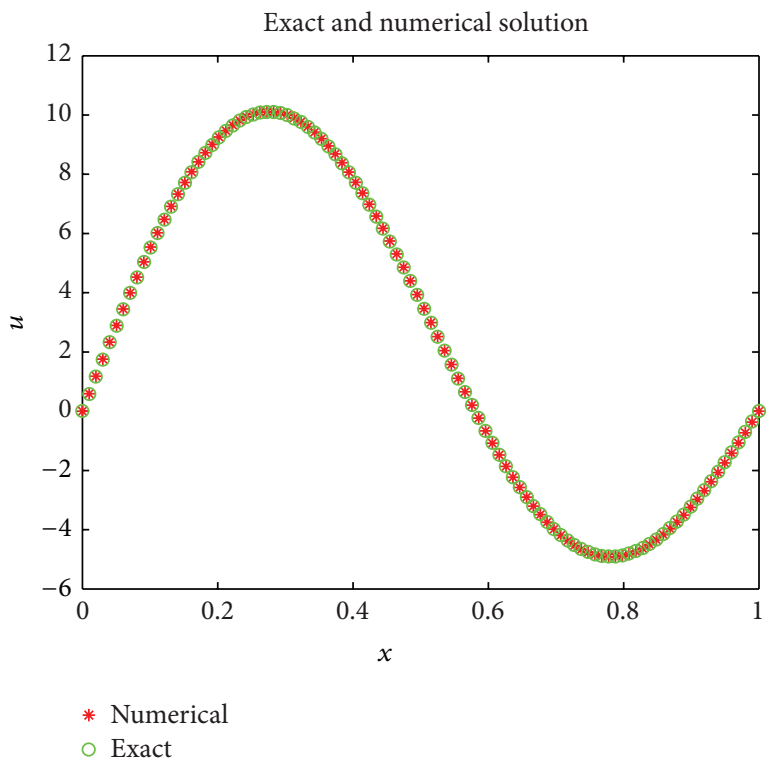

FIgure 4: Analytical and numerical solution at $t=2$, with $\mathrm{PH}$ as RBF, $\tau=0.1, d_{x}=0.05$, and $x \in[0,1]$, for Example 2 .

Remark 3. Note that, for the two examples shown previously, the RBFs are unconditionally positive definite to guarantee the solvability of the resulting systems. However, some RBFs are conditionally positive definite. These types of interpolation problems can be obtained by adding a polynomial term to the interpolation (12), and it is also easy to verify the efficiency of the proposed schemes for these cases.

\section{Conclusions}

In this paper we proposed and implemented the method of particular solutions to solve the one-dimensional timedependent Burgers' equation. The effectiveness of the computational scheme is well demonstrated. It must be emphasized

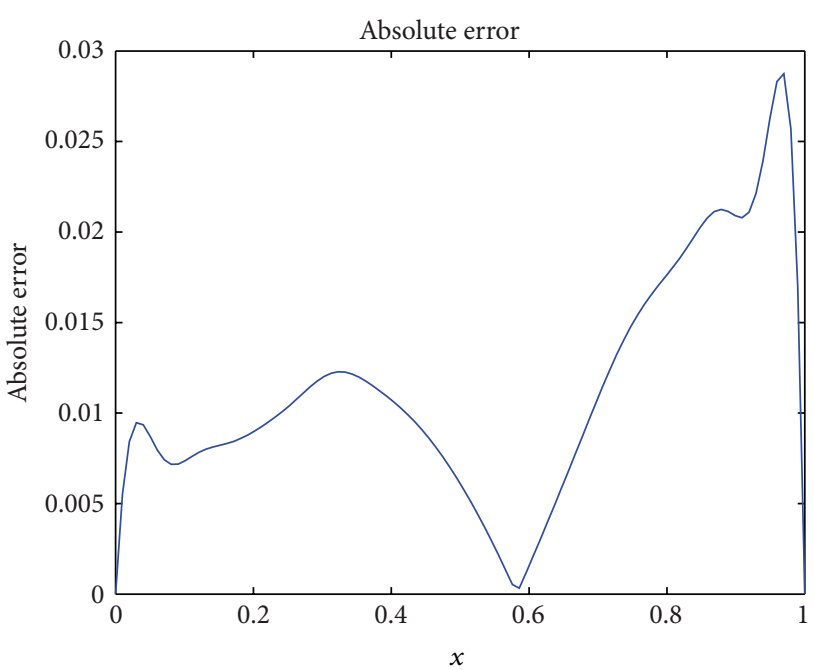

Figure 5: Absolute error at $t=2$, with PH as RBF, $\tau=0.1, d_{x}=$ 0.05 , and $x \in[0,1]$, for Example 2 .

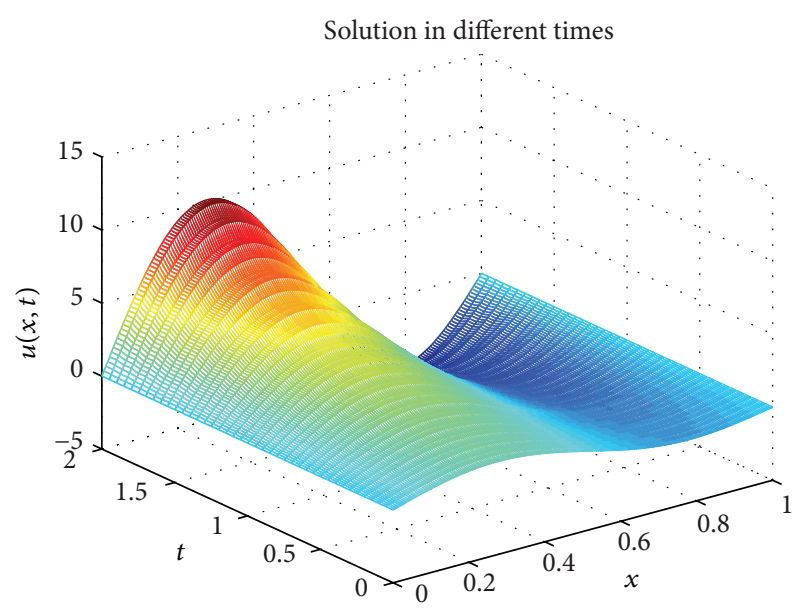

FIGURE 6: Space-time graph of the solution up to $t=2$, with $\mathrm{PH}$ as RBF, $\tau=0.1, d_{x}=\pi / 10$, and $x \in[0,2 \pi]$, for Example 2 .

that the choice of radial basis functions is a flexible feature of these methods. The radial basis functions can be globally supported, infinitely differentiable and contain free parameters, namely, shape parameters, which affect both accuracy of the solutions and conditioning of the collocation matrix. The optimal shape parameters in Examples 1 and 2 using IMQ and $\mathrm{PH}$, respectively, for all the calculations were found experimentally. The optimal choice of the shape parameters in RBFs is still an outstanding research problem [27-29]. A similar approach can be extended to solving $2 \mathrm{D}$ or $3 \mathrm{D}$ time-dependent partial differential equations. These research topics will be the focus of future investigation.

\section{Acknowledgments}

The work has been supported by the National Natural Science Foundation of China (Grants nos. 51190094, 61271337, 11201211, and 11201212) and AMEP of Linyi University. Special 
thanks are given to the reviewers for constructive advice and thoughtful comments.

\section{References}

[1] H. Bateman, "Some recent researches on the motion of fluids," Monthly Weather Review, vol. 43, no. 4, pp. 163-170, 1915.

[2] J. M. Burgers, "Mathematical examples illustrating relations occurring in the theory of turbulent fluid motion," vol. 17, no. 2, pp. 1-53, 1939.

[3] J. M. Burgers, "A mathematical model illustrating the theory of turbulence," Advances in Applied Mathematics, vol. 1, pp. 171199, 1948.

[4] J. D. Cole, "On a quasi-linear parabolic equation occurring in aerodynamics," Quarterly of Applied Mathematics, vol. 9, pp. 225-236, 1951.

[5] E. Hopf, "The partial differential equation $u_{t}+u u_{t}=\mu u_{x x}$," Communications on Pure and Applied Mathematics, vol. 3, pp. 201-230, 1950.

[6] E. R. Benton and G. W. Platzman, "A table of solutions of the one-dimensional Burgers equation," Quarterly of Applied Mathematics, vol. 30, pp. 195-212, 1972.

[7] I. A. Hassanien, A. A. Salama, and H. A. Hosham, "Fourthorder finite difference method for solving Burgers' equation," Applied Mathematics and Computation, vol. 170, no. 2, pp. 781800, 2005.

[8] S. Kutluay, A. Esen, and I. Dag, "Numerical solutions of the Burgers' equation by the least-squares quadratic B-spline finite element method," Journal of Computational and Applied Mathematics, vol. 167, no. 1, pp. 21-33, 2004.

[9] M. Dehghan and M. Tatari, "Determination of a control parameter in a one-dimensional parabolic equation using the method of radial basis functions," Mathematical and Computer Modelling, vol. 44, no. 11-12, pp. 1160-1168, 2006.

[10] M. Dehghan, "Finite difference procedures for solving a problem arising in modeling and design of certain optoelectronic devices," Mathematics and Computers in Simulation, vol. 71, no. 1, pp. 16-30, 2006.

[11] S. Chantasiriwan, "Cartesian grid methods using radial basis functions for solving Poisson, Helmholtz, and diffusionconvection equations," Engineering Analysis with Boundary Elements, vol. 28, no. 12, pp. 1417-1425, 2004.

[12] G. R. Liu and Y. T. Gu, "Boundary meshfree methods based on the boundary point interpolation methods," Engineering Analysis with Boundary Elements, vol. 28, no. 5, pp. 475-487, 2004.

[13] G. Fairweather and A. Karageorghis, "The method of fundamental solutions for elliptic boundary value problems," Advances in Computational Mathematics, vol. 9, no. 1-2, pp. 6995, 1998.

[14] A. S. Muleshkov, M. A. Golberg, and C. S. Chen, "Particular solutions of Helmholtz-type operators using higher order polyharmonic splines," Computational Mechanics, vol. 24, no. 5-6, pp. 411-419, 1999.

[15] C. S. Chen, C. M. Fan, and P. H. Wen, "The method of approximate particular solutions for solving elliptic problems with variable coefficients," International Journal of Computational Methods, vol. 8, no. 3, pp. 545-559, 2011.

[16] T. Jiang, M. Li, and C. S. Chen, "The method of particular solutions for solving inverse problems of a nonhomogeneous convection-diffusion equation with variable coefficients," Numerical Heat Transfer A, vol. 61, no. 5, pp. 338-352, 2012.
[17] T. Jiang, X. Wang, and Z. Zhang, "Solutions for one-dimensional time-dependent Schrodinger equations," in Information Computing and Applications, pp. 371-378, Springer, Berlin, Germany, 2012.

[18] T. Belytschko, Y. Y. Lu, and L. Gu, "Element-free Galerkin methods," International Journal for Numerical Methods in Engineering, vol. 37, no. 2, pp. 229-256, 1994.

[19] W. Chen, "Symmetric boundary knot method," Engineering Analysis with Boundary Elements, vol. 26, no. 6, pp. 489-494, 2002.

[20] M. D. Buhmann, Radial Basis Functions, Cambridge University Press, Cambridge, UK, 2003.

[21] K. E. Atkinson, "The numerical evaluation of particular solutions for Poisson's equation," IMA Journal of Numerical Analysis, vol. 5, no. 3, pp. 319-338, 1985.

[22] A. H.-D. Cheng, "Particular solutions of Laplacian, Helmholtztype, and polyharmonic operators involving higher order radial basis functions," Engineering Analysis with Boundary Elements, vol. 24, no. 7-8, pp. 531-538, 2000.

[23] H. A. Cho, M. A. Golberg, A. S. Muleshkov, and X. Li, "Trefftz methods for time dependent partial differential equations," Computers, Materials and Continua, vol. 1, no. 1, pp. 1-38, 2004.

[24] S. Yu. Reutskiy, C. S. Chen, and H. Y. Tian, "A boundary meshless method using Chebyshev interpolation and trigonometric basis function for solving heat conduction problems," International Journal for Numerical Methods in Engineering, vol. 74, no. 10, pp. 1621-1644, 2008.

[25] X. Wang, T. Jiang, and Z. Jiang, "PDEs solution based on two-stage MFS-MPS and onestage MFS-MPS," in Information Computing and Applications, pp. 356-363, Springer, Berlin, Germany, 2012.

[26] C. A. Micchelli, "Interpolation of scattered data: distance matrices and conditionally positive definite functions," Constructive Approximation, vol. 2, no. 1, pp. 11-12, 1986.

[27] S. Rippa, "An algorithm for selecting a good value for the parameter $c$ in radial basis function interpolation," Advances in Computational Mathematics, vol. 11, no. 2-3, pp. 193-210, 1999.

[28] J. Wertz, E. J. Kansa, and L. Ling, "The role of the multiquadric shape parameters in solving elliptic partial differential equations," Computers \& Mathematics with Applications, vol. 51, no. 8, pp. 1335-1348, 2006.

[29] H. Xie and D. Li, "A meshless method for Burgers' equation using MQ-RBF and highorder temporal approximation," Applied Mathematical Modelling, 2013. 


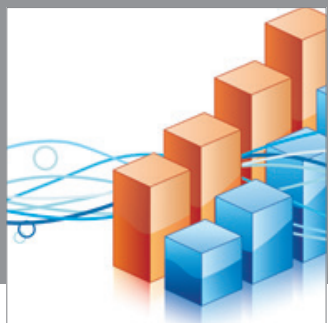

Advances in

Operations Research

mansans

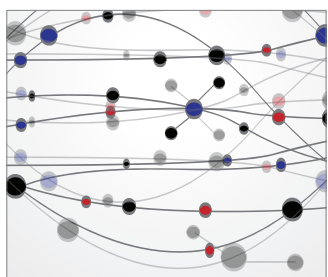

The Scientific World Journal
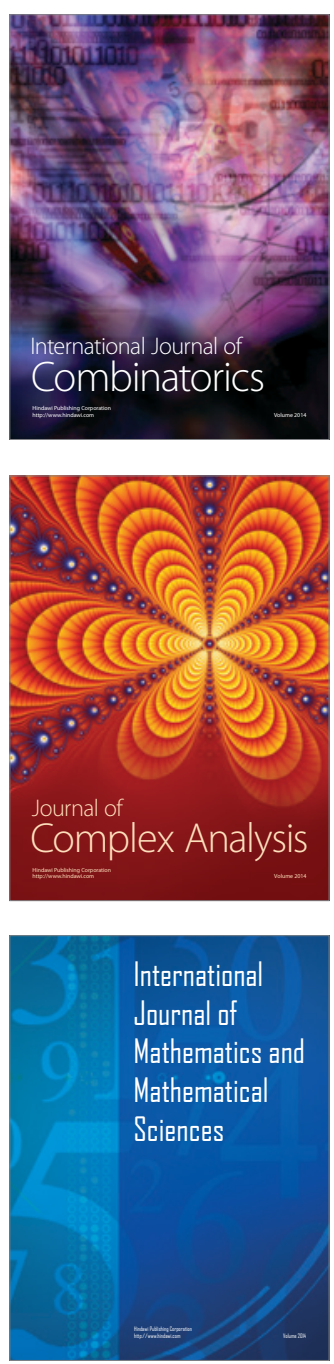
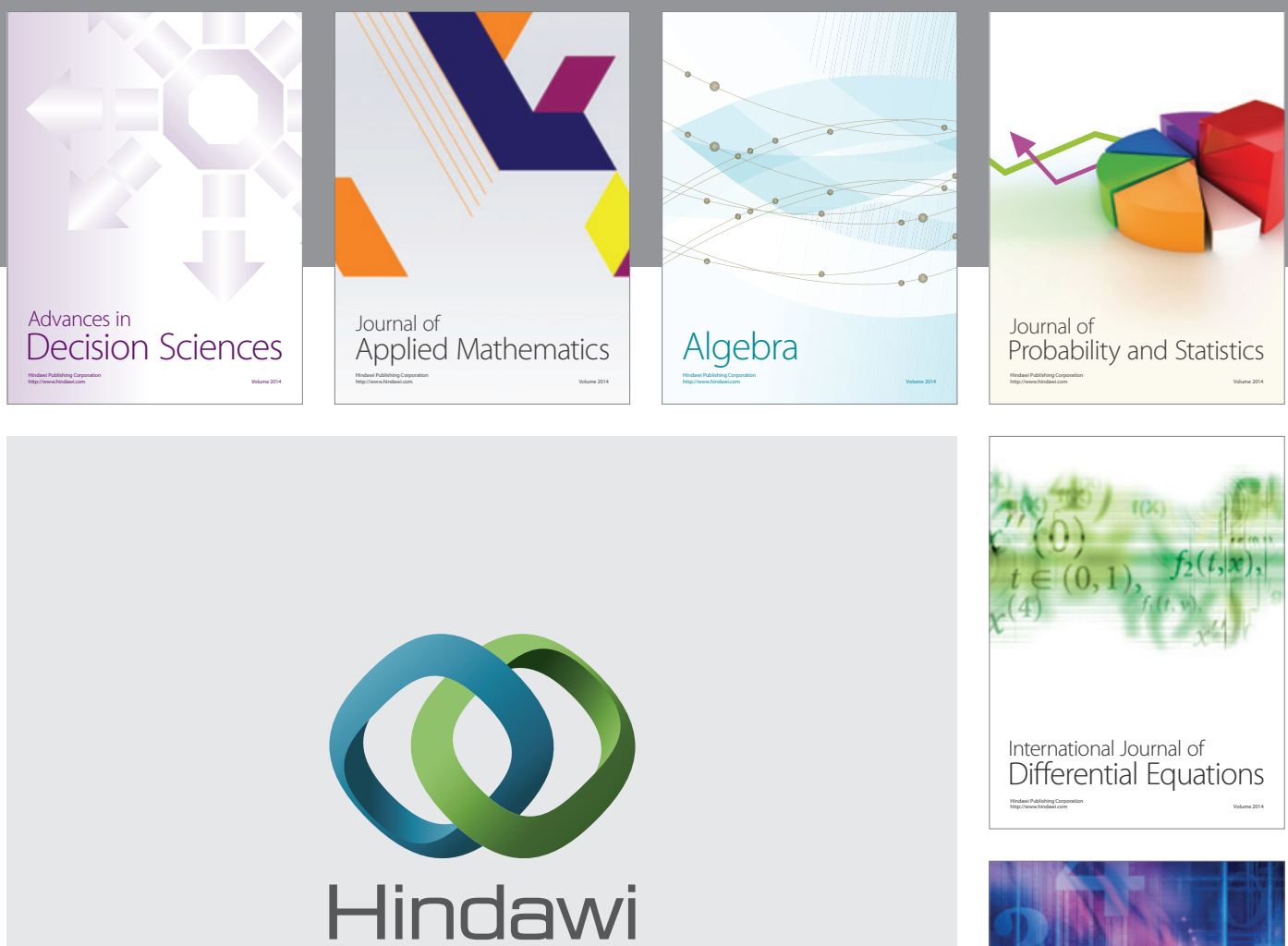

Submit your manuscripts at http://www.hindawi.com
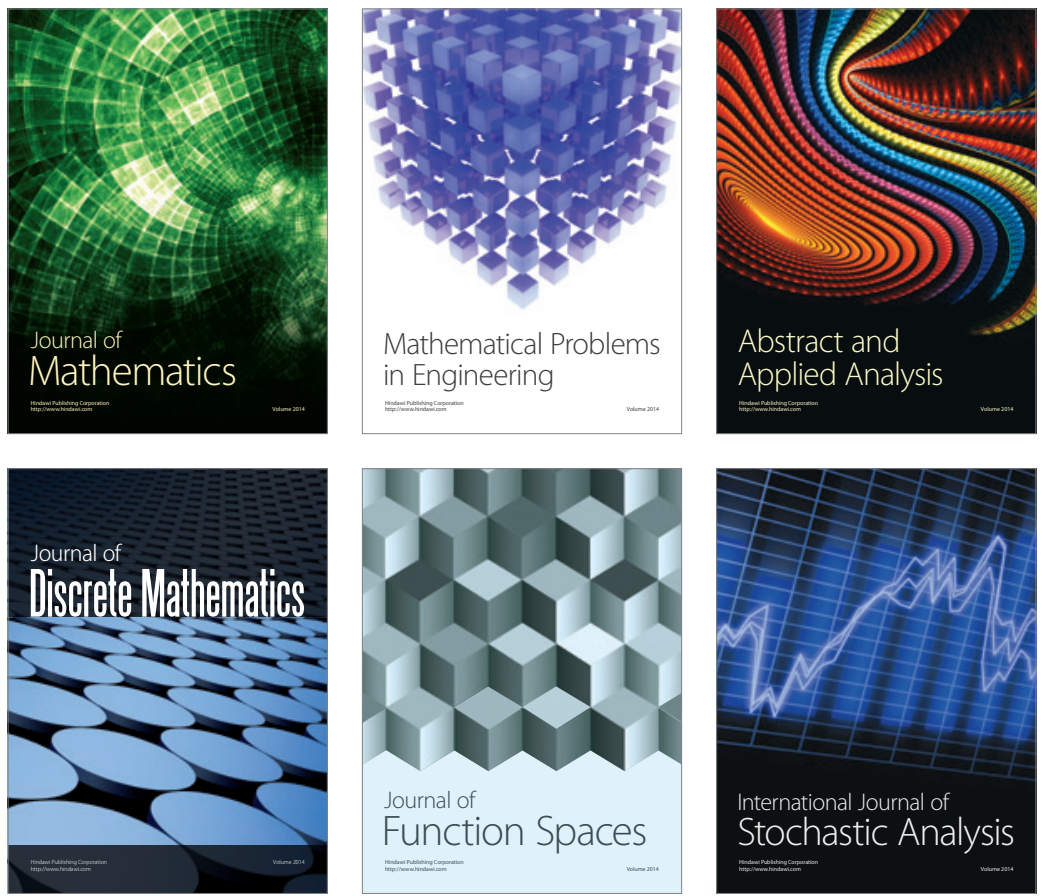

Journal of

Function Spaces

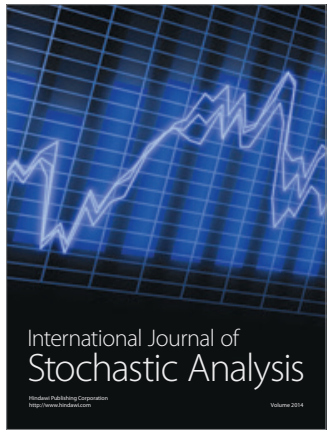

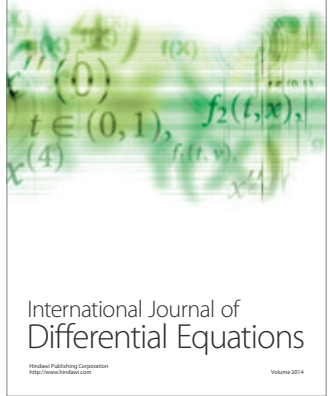
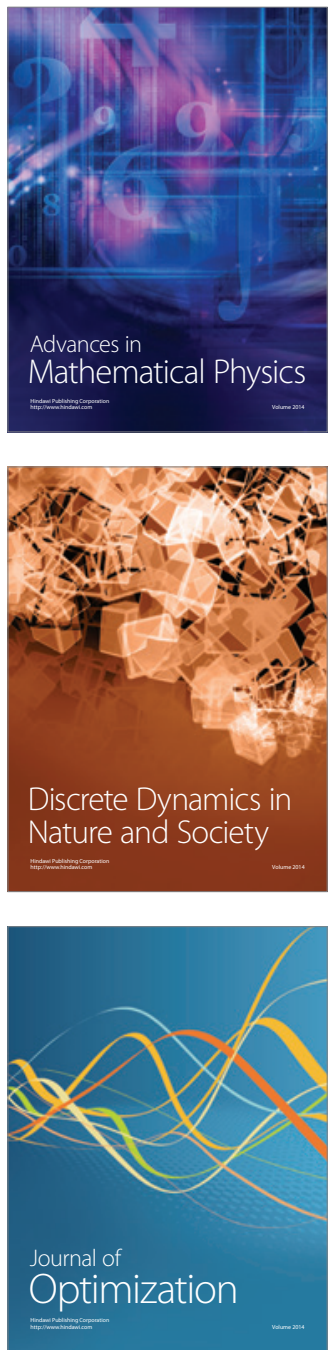